\title{
Application of genetic algorithm in extracting cell dielectric characteristics with electrorotation
}

\author{
Elnaz Alizadeh-Haghighi, Samad Jafarmadar, Shahram Khalilarya
}

Mechanical Engineering Department, Urmia University, Urmia, Iran

E-mail any correspondence to: e.alizadeh@urmia.ac.ir

\begin{abstract}
In this study the transformed theory is applied to derive the dielectric characteristics of cells, considering the electrorotation (ER) peak frequency. In current studies, estimations of low frequency, which are credible for the values less than $1 \mathrm{mS} / \mathrm{m}$ for medium conductivity, are used to obtain the corresponding permittivity and conductivity of cells. Unlike the presented works, the transformed theory applies the comprehensive statement for corresponding permittivity and conductivity of cells. In the transformed theory, the membrane and interior characteristics could be obtained from the high and the low frequencies of peak $E R$, for all values of conductivity of medium. Characteristics of cells are obtained via optimization of an equation for the conductivity of medium regarding the peak ER frequency. The optimization process is performed applying genetic algorithm due to its swift adaptation to the problem and faster convergence.
\end{abstract}

Keywords: Electrorotation, genetic algorithm, cell dielectric properties, interior permittivity, membrane conductivity

\section{Introduction}

When subjected to magnetic or electric fields all particles experience torques and forces since they have magnetic and electrical properties related with their containing materials and their shape. Furthermore, due to the particles own electrical charge, magnetization or polarization, they will reveal quite strong mutual interactions. The study of torques and forces exerted on tiny particles (less than approximately $10^{3}$ micro-meters in diameter) and also the dynamics and mechanics induced by these torques and forces through the action of a magnetic or electric field, is known as particle electromechanics. Lab- on-a-chip (LOC) devices which apply electromechanics, have recently become very attractive due to pursuing development of portable, individualized, and implantable devices for point-of-care applications [1]. Moreover, LOC devices reveal unique advantages such as, being cost effective, need for very small amount of sample, high level of automation, and high throughput [2].

LOC devices apply electrorotation (ER), dielectrophoresis (DEP) and traveling wave dielectrophoresis (twDEP) broadly. All of the mentioned phenomena are created due to the particle induced dipole moment interaction with the electric field. Generalized dielectrophoresis is a suitable title for discussing DEP, ER, and twDEP together in a simultaneous and systematical way [3].

ER is used extensively in manipulation and characterization of particles/cells and is the most accurate method for dielectric characterization among the mentioned phenomena in the literature. It reveals membrane and cells' interior dielectric properties using the frequencydependent rotational responses. ER is a noninvasive technique, which can be used for finding changes in the physicochemical and morphology properties of microorganisms [4]. Applying ER, the influences of different biocide doses on yeast cell suspensions have been studied [5]. The specific membrane capacitance value was obtained by Sukhorukov observing rotation of single cells induced by a homogeneous rotating field [6]. An essential effort in cell studies is to derive dielectric characteristics of cells from the ER experimental results. In these experiments, the cells inside a medium with permittivity $\varepsilon_{m}$ and conductivity $\sigma_{m}$ are exposed to an AC 
field, which rotates uniformly [7]. One of the major analyzes in these ER experiments is to determine the frequency where the rate of electrorotation turn into its maximum. This frequency is known as the peak frequency $f_{p k}$. Since the full expressions for the cell's effective dielectric properties in terms of $f_{p k}$ are nonlinear equations, they cannot be solved analytically [8]. In order to achieve the equivalent cell permittivity $\varepsilon_{p}$ and conductivity $\sigma_{p}$ estimations of low frequency were used by Schwan [9]. In the ER experiments with convenient medium conductivity, a couple of peak frequencies may occur. It was suggested to use the high frequency limit for the higher peak frequency [3]. In this case, the effective cell permittivity and conductivity can be considered as the cytoplasm permittivity and conductivity, respectively. The estimation of low frequency is useful in the case of measurement inside a less conductive medium and where the peak frequency is adequately low. Furthermore, in the experiments with a higher peak frequency like $100 \mathrm{MHz}$ these estimations are invalid for attaining the dielectric characteristics of cells [10]. Thus, this theory is in most situations not suitable for cell dielectric characterization from measured data. Therefore, it is essential to study the cases when the estimations of low frequency would suffer from inefficiency. Discovering the effective substitution for the mentioned theory to investigate the dielectric characteristics of cells using the ER measurements is an essential issue [11].

In this paper, the transformed method in which the comprehensive statements for corresponding permittivity and conductivity of cells are used, is applied [11]. Since the current optimization process cannot be used directly for a peak frequency's nonlinear equation, a distinct method was applied in this study to obtain the cell dielectric characteristics [12]. The mentioned transformed theory is credible for the high and the low peak frequencies. On the other hand this method is applicable for all values of conductivity of the medium [11]. In order to achieve the cell dielectric properties in this study, genetic algorithm (GA) is applied as a robust means to optimize the equation of modified theory due to its capacity for quick adaptation to the problem and faster convergence without requiring derivative information.

\section{Theory}

\section{Inhomogeneous particle modeling}

Living particles like cells are heterogeneous and a multi shell model could take their heterogeneous structures into account [13]. In order to describe non-spherical shells and the plasma membrane dielectric anisotropy, the extended form of this model has been used [14,15].
Maxwell-Wagner polarizations will occur where the various shells join. The temporal dynamics and degrees of these diverse polarizations relate to the dielectric characteristics of the shells constitutive materials.

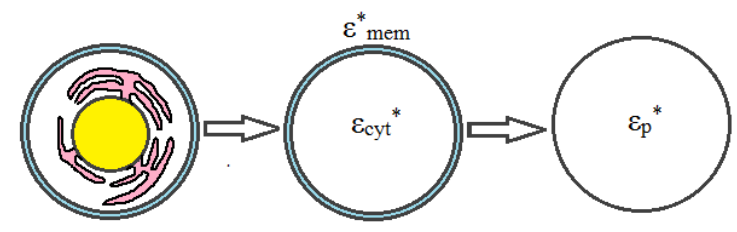

Figure 1. Continuous simplification of a cell to a homogeneous particle of equivalent permittivity $\varepsilon_{p}^{*}$. The cell is represented as cytoplasm covered by a membrane with complex permittivities $\varepsilon_{\text {cyt }}^{*}$ and $\varepsilon_{\text {mem }}^{*}$, respectively [16].

For a multi shell particle, the Clausius Mossotti function could be achieved by investigating the particle's effective complex permittivity $\varepsilon_{p}^{*}$ and conductivity $\sigma^{*}$. In order to demonstrate that a multishell heterogeneous particle may be replaced hypothetically with a homogeneous particle, the word effective is applied. The electric field around the particle would not be changed by the replacement of a heterogeneous particle with the mentioned homogeneous one. Fig. 1 demonstrates the cell simplification steps to an uncomplicated, homogeneous particle. Huang et al. formulated a method that employs equations adaptable to computer modeling [17]. The complex effective permittivity of human erythrocyte that can be modeled by the single-shell model represented as

$$
\begin{aligned}
& \varepsilon_{p}^{*}=\varepsilon_{p}^{*}-j \sigma_{p} \\
& =\varepsilon_{m e m}^{*}\left(\frac{(R /[R-d])^{3}+2\left(\frac{\varepsilon_{i n t}^{*}-\varepsilon_{m e m}^{*}}{\varepsilon_{i n t}^{*}+2 \varepsilon_{m e m}^{*}}\right)}{(R /[R-d])^{3}-\left(\frac{\varepsilon_{i n t}^{*}-\varepsilon_{m e m}^{*}}{\varepsilon_{i n t}^{*}+2 \varepsilon_{m e m}^{*}}\right)}\right)
\end{aligned}
$$

in which $\varepsilon_{p}$ and $\sigma_{p}$ represent the particle's effective permittivity and conductivity respectively. $\varepsilon^{*}$ int is the cytoplasm or interior's complex permittivity, $\varepsilon^{*}$ mem is the membrane's complex permittivity, $R$ represents the cell's outer radius, and $d$ shows the membrane's thickness.

For the majority of mammalian cells $d$ is three orders less than $R$, and so Eq. (1) can be simplified to the below form under the conditions $\frac{d}{R} \ll 1, \varepsilon_{m e m} \leq \varepsilon_{\text {int }}$, and , $\sigma_{m e m} \leq \sigma_{\text {int }}[8]$,

$$
\varepsilon_{p}^{*}=\varepsilon_{m e m}^{*}\left(\frac{\varepsilon_{i n t}^{*}}{\varepsilon_{\text {mem }}^{*}+\left(\frac{d}{R}\right) \varepsilon_{i n t}^{*}}\right)
$$

Eqs. (3) and (4) show the real part and the imaginary part of Eq. (2) respectively 


$$
\begin{aligned}
& \frac{\varepsilon_{p}^{*}}{\varepsilon_{p}^{*}}=\frac{\left(\frac{\varepsilon_{m e m}}{\varepsilon_{0}} \frac{\varepsilon_{\text {int }}}{\varepsilon_{0}}-\frac{\sigma_{m e m} \sigma_{i n t}}{\omega^{2} \varepsilon_{0}^{2}}\right)\left(\frac{\varepsilon_{m e m}}{\varepsilon_{0}}+\frac{d}{R} \frac{\varepsilon_{i n t}}{\varepsilon_{0}}\right)}{\left(\frac{\varepsilon_{m e m}}{\varepsilon_{0}}+\frac{d}{R} \frac{\varepsilon_{\text {int }}}{\varepsilon_{0}}\right)^{2}+\frac{1}{\omega^{2} \varepsilon_{0}^{2}}\left(\sigma_{m e m}+\frac{d}{R} \sigma_{\text {int }}\right)^{2}}+\frac{\frac{1}{\omega^{2} \varepsilon_{0}^{2}}\left(\sigma_{m e m} \frac{\varepsilon_{i n t}}{\varepsilon_{0}}-\frac{\varepsilon_{m e m}}{\varepsilon_{0}} \sigma_{\text {int }}\right)\left(\sigma_{m e m}+\frac{d}{R} \sigma_{\text {int }}\right)}{\left(\frac{\varepsilon_{\text {mem }}}{\varepsilon_{0}}+\frac{d}{R} \frac{\varepsilon_{\text {int }}}{\varepsilon_{0}}\right)^{2}+\frac{1}{\omega^{2} \varepsilon_{0}^{2}}\left(\sigma_{m e m}+\frac{d}{R} \sigma_{\text {int }}\right)^{2}} \\
& \sigma_{p}=\frac{-\left(\frac{\varepsilon_{m e m}}{\varepsilon_{0}} \frac{\varepsilon_{i n t}}{\varepsilon_{0}}-\frac{\sigma_{m e m} \sigma_{i n t}}{\omega^{2} \varepsilon_{0}^{2}}\right)\left(\sigma_{m e m}+\frac{d}{R} \sigma_{i n t}\right)}{\left(\frac{\varepsilon_{m e m}}{\varepsilon_{0}}+\frac{d}{R} \frac{\varepsilon_{i n t}}{\varepsilon_{0}}\right)^{2}+\frac{1}{\omega^{2} \varepsilon_{0}^{2}}\left(\sigma_{m e m}+\frac{d}{R} \sigma_{i n t}\right)^{2}}+\frac{\left(\sigma_{m e m} \frac{\varepsilon_{\text {int }}}{\varepsilon_{0}}-\frac{\varepsilon_{m e m}}{\varepsilon_{0}} \sigma_{\text {int }}\right)\left(\frac{\varepsilon_{m e m}}{\varepsilon_{0}}+\frac{d}{R} \frac{\varepsilon_{\text {int }}}{\varepsilon_{0}}\right)}{\left(\frac{\varepsilon_{m e m}}{\varepsilon_{0}}+\frac{d}{R} \frac{\varepsilon_{\text {int }}}{\varepsilon_{0}}\right)^{2}+\frac{1}{\omega^{2} \varepsilon_{0}^{2}}\left(\sigma_{m e m}+\frac{d}{R} \sigma_{\text {int }}\right)^{2}} \\
& \frac{\varepsilon_{p}}{\varepsilon_{0}}=\frac{\left(\frac{\varepsilon_{m e m}}{\varepsilon_{0}}\right)^{2}\left(\frac{\varepsilon_{i n t}}{\varepsilon_{0}}\right)+\frac{\sigma_{i n t}^{2}}{\omega^{2} \varepsilon_{0}^{2}}\left(\frac{\varepsilon_{m e m}}{\varepsilon_{0}}\right)\left(\frac{d}{R}\right)}{\left(\frac{\varepsilon_{m e m}}{\varepsilon_{0}}\right)^{2}+\frac{\sigma_{\text {int }}^{2}}{\omega^{2} \varepsilon_{0}^{2}}\left(\frac{d}{R}\right)^{2}}+\text { terms of three orders less } \\
& \sigma_{p}=\frac{\left(\frac{\sigma_{m e m} \sigma_{i n t}^{2}}{\omega^{2} \varepsilon_{0}^{2}}\right)\left(\frac{d}{R}\right)+\left(\frac{\varepsilon_{m e m}}{\varepsilon_{0}}\right)^{2} \sigma_{i n t}}{\left(\frac{\varepsilon_{m e m}}{\varepsilon_{0}}\right)^{2}+\frac{\sigma_{i n t}^{2}}{\omega^{2} \varepsilon_{0}^{2}}\left(\frac{d}{R}\right)^{2}}+\text { terms of three orders less }
\end{aligned}
$$

The terms in Eqs. (3) and (4) can be classified into terms with factor $1 / \omega^{2}$ and terms without this factor. Eqs. (3) and (4) reduce to the forms of Eqs. (5) and (6) keeping each group's basic term in the numerator and the denominator [11].

Eqs. (5) and (6) are applicable for all angular frequencies, $\omega$. Since the leading term is of two orders greater than the term needed for obtaining the conductance of membrane, the terms of three orders less than the leading term are omitted [11].

\section{Transformed Theory}

In this part, a transformation of the current theory is presented for the cases where the estimation of low frequency limit is not applicable for obtaining the characteristics of cell from the investigations of ER peak frequency. In order to indicate the equivalent permittivity $\varepsilon_{p}$ and conductivity $\sigma_{p}$, in Eqs. (1), the simplified form of the full expressions Eqs. (3) and (4) are employed in the modified theory [11]. Afterwards the nonlinear algebraic equations for $f_{p k}$ is achieved. These equations are implicit equations in terms of cell features, medium permittivity and medium conductivity $\sigma_{\mathrm{m}}$ [11]. The cell characteristics were obtained applying an optimization method to the nonlinear equations, which are mentioned above [12]. The equation for the peak rotational angular frequency $\omega_{\mathrm{pk}}$ and particle/medium dielectric properties is presented below. This equation is obtained setting the derivation of the Clausius Mossotti factor to zero.

$$
\omega_{p k}=2 \pi f_{p k}=\left(\frac{\sigma_{p}+2 \sigma_{m}}{\varepsilon_{p}+2 \varepsilon_{m}}\right)
$$

where $f_{p k}$ is the peak frequency, $\varepsilon_{m}$ is the effective medium permittivity and $\sigma_{m}$ represents the effective medium conductivity.

Expressing the Eq. (7) explicitly is the essential step in finding the cell characteristics through optimization. Since an explicit solution of $f_{p k}$ from the Eq. (7) cannot be gained, an explicit solution for $\sigma_{m}$ in terms of $f_{p k}$ is used instead. The below equation, which is applied in the modified theory, is obtained by rewriting the Eq. (7) for medium conductivity [11]:

$$
\sigma_{m}=\frac{1}{2}\left\{2 \pi f_{p k}\left(\varepsilon_{p}+2 \varepsilon_{m}\right)-\sigma_{p}\right\}
$$

As a result, after the shift between the independent and dependent variable's roles, the optimization is applied. According to Eqs. (3) and (4), (5) and (6), $\varepsilon_{p}$ and $\sigma_{p}$ are not functions of $\sigma_{\mathrm{m}}$, but they both are functions of $\omega$ and the cell properties. Therefore, Eq. (8) is an explicit equation for $\sigma_{m}$.

Equations (8) is represented as a functional type as bellow [11].

$$
\begin{aligned}
& \sigma_{m}=\sigma_{m, \text { theory }} \\
& =\sigma_{m}\left(R f_{p k}, R, \varepsilon_{m}, d / R, \sigma_{m e m}, \varepsilon_{m e m}, \sigma_{\text {int }}, \varepsilon_{\text {int }}\right)
\end{aligned}
$$

The number of variables in Eq. (9) is more than that in the recently used theory since the more complete expressions for $\varepsilon_{p}$ and $\sigma_{p}$ shown by Eqs. (5) and (6) are used here. In the present study, $d / R$ and $\varepsilon_{m}$ are assumed to be 1000 and $80 \varepsilon_{0}$, respectively. $f_{p k}$ and $R$ are obtained through the experiments, and the parameters $\sigma_{\text {mem }}, \varepsilon_{\text {mem }}, \sigma_{\text {int, }}$ and $\varepsilon_{\text {int }}$ could be obtained via the optimization. This optimization is performed by minimizing the below variance [11], 


$$
\Delta \sigma_{m}=\sum_{i=1}^{N}\left(\sigma_{m, \text { theory }}-\sigma_{m, \exp }\right)^{2}
$$

where applying Eq. (8) would lead to the calculation of $\sigma_{\mathrm{m} \text {,theory. }}$ The medium conductivity $\sigma_{\mathrm{m} \text {,exp }}$ is obtained from experiment. The measured data set number, $\sigma_{\mathrm{m}}$ and $R f_{p k,}$ is shown by $\mathrm{N}$.

In order to optimize Equation (10), GA is applied in this study.

\section{Genetic Algorithm}

One stochastic method for general search and optimization is genetic algorithm (GA), which is inspired by the biological world. Holland first proposed GA in 1975 [18]. GA is a population based, iterative, parallel global search algorithm and has been used in many scientific areas such as optimization problems and was found to be very efficient [19]. The search is performed by simulating evolution in which more successful organisms in nature appear. Natural selection, which means survival of the fittest is the main idea of GAs. A complicated, long thread of DNA (deoxyribonucleic acid) is called chromosome. Individual's particular features are determined along these chromosomes. Features are coded by some DNA combination. During reproduction, changes occur in chromosomes. In the crossover process the parents' chromosomes randomly change thus, the offspring reveals some features of each parent. In addition, some traits are changed during a rarer process called mutation. All the parameters can be altered simultaneously in the GA method; therefore, it can obtain a faster convergence [20].

In this work, a random population was produced to use as the initial population. Then considering the function value of the individual, the fitness was evaluated and best individuals were utilized as parents of the next generation. Genetic transformation such as crossover and mutation applied on these individuals. Two individuals are randomly selected as parents in a crossover process and part of their construction are exchanged to form two new individuals. In the mutation process, one individual is randomly selected from the parent population and its internal representation is changed. In order to improve search performance both of the crossover and mutation rates should be carefully chosen. The produced child population turned to the parent population for the next generation and undergoes the same process until termination conditions, which were determined as stopping criteria, has been satisfied.

The parameters that should be specified in using GA are the number of the initial population and the maximum generation, upper and lower bounds of genes (variables), the chromosomes' length, the crossover and the mutation probability. The algorithm achieves best results when the values for the first two parameters rise. Nine experimental data sets for medium conductivity and peak rotational frequency obtained by Pethig et al. for insulin secreting cells (primary $\beta$-cells and INS- 1 insulinoma cells) are applied in optimization in this work [7].

\begin{tabular}{cc}
$\begin{array}{l}\text { Table 1. Experimental data sets for medium conductivity and } \\
\text { peak rotational frequency. }\end{array}$ \\
\hline \begin{tabular}{cc} 
Medium conductivity & Peak rotational frequency \\
$\sigma_{\mathrm{m}}(\mathrm{mS} / \mathrm{m})$ & $\omega_{\mathrm{pk}}(\mathrm{MHz})$ \\
\hline 11.5 & 0.46 \\
21.7 & 0.70 \\
32.8 & 1.16 \\
42.5 & 1.53 \\
48.7 & 2.01 \\
60.3 & 2.20 \\
74.9 & 2.62 \\
86.9 & 3.17 \\
101.4 & 3.62
\end{tabular}
\end{tabular}

For human erythrocytes a radius of equilibrium $2.62 \mu \mathrm{m}$ and $d=5 \mathrm{~nm}, d / R=1.91 \times 10^{-3}$ is used in this work. In the literature, the following assumptions are used for human erythrocytes and other mammalian cells [21].

$$
\begin{gathered}
\frac{\varepsilon_{m e m}}{\varepsilon_{0}} \approx 10, \\
\sigma_{m e m} \approx 10^{-6} \frac{S}{m} \\
\frac{\varepsilon_{i n t}}{\varepsilon_{0}} \approx 10^{2}, \\
\sigma_{i n t} \approx 1 \frac{S}{m}, \\
d / R \approx 10^{-3}
\end{gathered}
$$

The best fitness with the initial population of 60 and the number of generations of 51 was obtained by trial and error. After setting parameters of genetic algorithm in Matlab, the values of fitness vs generation number are shown in Fig. 2.

The mean fitness values are demonstrated in every generation by the points on top of the plot, while the bottom points illustrate the value of the best fitness. The values of the best and mean fitness in 51th generation are equal to $4.7952 \times 10^{-4}$ and $4.7956 \times 10^{-4}$, respectively. The values of the best fitness' change decrease at last generations, since the best point is getting closer.

In order to reach optimal cell properties the GA was run many times. Considering the end populations of different runs, the best results for the cell property were demonstrated in Table 2. 


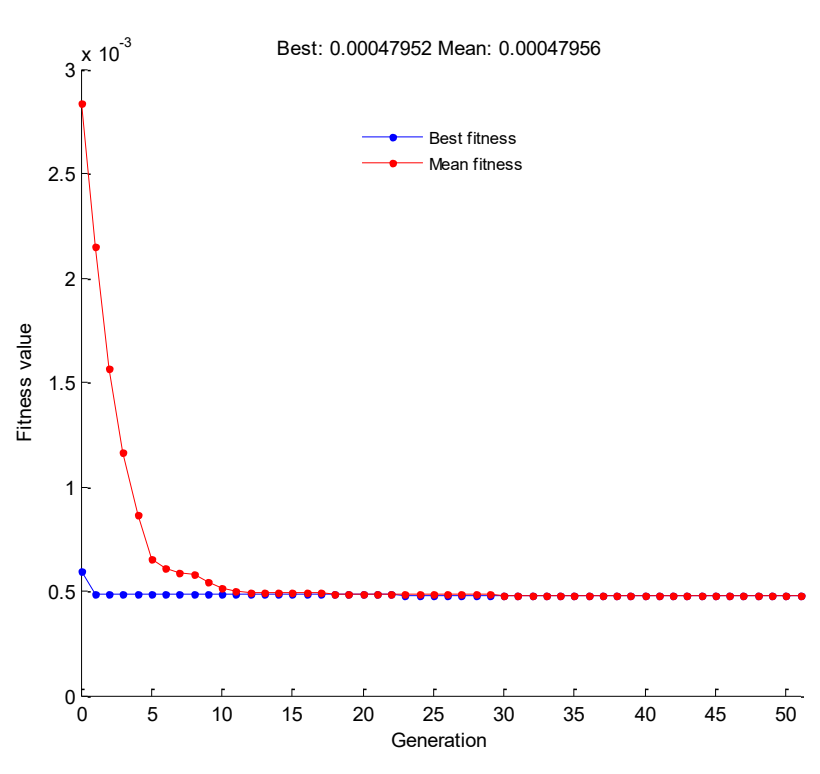

Figure 2. The fitness values of a hold signal vs number of generations.

Table 2: the best results for cell properties obtained from genetic optimization

\begin{tabular}{ll}
\hline Cell Property & Value \\
\hline Membrane Conductivity $\sigma_{\text {mem }}$ & $7.043 \times 10^{-7} \mathrm{~S} / \mathrm{m}$ \\
Membrane Permittivity $\varepsilon_{m e m}$ & $1 \times 10^{-10} \mathrm{~F} / \mathrm{m}$ \\
Interior Conductivity $\sigma_{\text {int }}$ & $1.3 \mathrm{~S} / \mathrm{m}$ \\
Interior Permittivity $\varepsilon_{\text {int }}$ & $1.507 \times 10^{-9} \mathrm{~F} / \mathrm{m}$ \\
\hline
\end{tabular}

\section{Conclusion}

The dielectric properties of cells were obtained applying low frequency approximations. However, transformed theory could be employed in the case the estimations of low frequency are not convenient to obtain the effective permittivity and conductivity of the cell. The transformed theory that is used in this study can lead to logical results for electrorotation experiments performed at the medium with high conductivities. In this theory obtaining dielectric characteristics of cell at every value of medium conductivity using both the high and the low electrorotation peak frequency is possible. Extracting the characteristics of interior and membrane of the cell by measuring electrorotation peak frequency is accomplished in this work. The optimization on medium conductivity's function in term of peak frequencies resulted in the cell interior and membrane conductivity and permittivity values. GA algorithm is an efficient optimization method, which is used in this study due to its ability of fast convergence and adaptation to the problem.

\section{References}

1. Kost, G. J. Principles and Practice of Point-of-care Testing. Philadelphia: Lippincott Williams and Wilkins. 2002; Chapter 1: 3-12.
2. Erickson, D, Li, D. Integrated microuidics devices, Analytica Chimica Acta. 2004; 149: 11-26. https://doi.org/10.1016/j.aca.2003.09.019

3. Lei, U, Lo, Y.J. Review of the theory of generaliseddielectrophoresis, IET Nanobiotechnology. 2011; 5(3): 86. https://doi.org/10.1049/iet-nbt.2011.0001

4. Dalton, C, Goater, A. D, Pething, R, Smith, H. V. Viability of Giardia intestinalis Cysts and Viability an Sporulation State of Cyclosporacayetanensis Oocysts Determined by Electrorotation, Applied and Environmental Microbiology, 2001; 586-590

5. Zhou, XF, Markx, GH, Pethig, R. Effect of biocide concentration on electrorotation spectra of yeast cells, Biochim. Biophy. Acta. 1996; 1281(1): 60-4.

6. Sukhorukov, V.L, Zimmermann, U. Rotating-Field-Induced Rotation and Measurement of the Membrane Capacitance of Single Mesophyll Cells of A vena sativa, J. Membrane Biol. 1993; 132: 27-40.

7. Pethig, R, Jakubek, L, Sanger, R.H, Heart, E, Corson, E, Smith, P.J.S. Electrokinetic measurements of membrane capacitance and conductance for pancreatic $\beta$-cells, IEE Proc. Nanobiotechnology. 2005; 152(6): 89-193.

8. Jones, T. B. Electromechanics of Particles, Cambridge University Press, Cambridge. 1995. https://doi.org/10.1017/СBO9780511574498

9. Arnold, W M, Zimmermann, U. Electro-rotation: Developments of a technique for dielectric measurements on individual cells and particles, J. Electrostatics. 1988; 21: 151-191. https://doi.org/10.1016/0304-3886(88)90027-7

10. Chung, C, Waterfall, M, Pells, S, Menachery, A, Smith, S, Pethig, R. Dielectrophoretic characterization of mammalian cells above $100 \mathrm{MHz}$, J. Electr. Bioimp. 2011; 2: 64-71. https://doi.org/10.5617/jeb.196

11. Lei, $U$, Sun, P-H, Pethig, R. Refinement of the theory for extracting cell dielectric properties from dielectrophoresis and electrorotation experiments. Biomicrofluidics. 2011; 5 (44109). https://doi.org/10.1063/1.3659282

12. Huang, $Y$, Wang, $X$ B, Becker, F F, Gascoyne, P R. Membrane changes associated with the temperature-sensitive P85gagmos-dependent transformation of rat kidney cells as determined by dielectrophoresis and electrorotation. Biochim. Biophys. Acta, 1996; 1282: 76-84. https://doi.org/10.1016/0005-2736(96)00047-8

13. Irimajiri, A, Hanai, T, Inouye, A. A dielectric theory of "multistratified shell" model with its application to a lymphoma cell. J Theor Biol. 1979; 21;78(2): 251-269.

14. Kakutani, T, Shibatani, S, Sugai, M. Electrorotation of nonspherical cells: theory for ellipsoidal cells with an arbitrary number of shells. Bioelectrochem. Bioenerg. 1993; 31: 131. https://doi.org/10.1016/0302-4598(93)80002-C

15. Sukhorukov, V. L, Meedt, G, Kurschner, M, Zimmermann, U. A single-shell model for biological cells extended to account for the dielectric anisotropy of the plasma membrane. J.

Electrost. 2001; 50: 191-204. https://doi.org/10.1016/\$0304-3886(00)00037-1 
16. Pethig, R. Dielectrophoresis: An assessment of its potential to aid the research and practice of drug discovery and delivery, Advanced Drug Delivery Reviews. 2013; 65: 15891599. https://doi.org/10.1016/j.addr.2013.09.003

17. Huang, $Y$, Holzel, R, Pethig, R, Wang, $X-B$, Differences in the $A C$ electrodynamics of viable and non-viable yeast cells determined through combined dielectrophoresis and electrorotation studies, Phys. Med. Biol. 1992; 37(7): 14991517. https://doi.org/10.1088/0031-9155/37/7/003

18. Holland, J. H. Adaptation in Natural and Artifical Systems, University of Michigan Press, Michigan.1975.
19. Goldberg, D. E. Genetic algorithm in search, optimization and machine learning. Reading MA Addison Wesley. 1989.

20. Safak, H, Sahin, M, Gulveren, B, Tomak, M. Efficiency of genetic algorithm and determination of ground state energy of impurity in a spherical quantum dot. Int. J. Mod. Phys. C, 2003; 14: 775-784. https://doi.org/10.1142/S0129183103004917

21. Gimsa, J, Müller, T, Schnelle, T, and Fuhr, G. Dielectric spectroscopy of single human erythrocytes at physiological ionic strength: dispersion of the cytoplasm. Biophys. J. 1996; 71: 495-506.

https://doi.org/10.1016/S0006-3495(96)79251-2 